which was finally lost sight of; but a complete ivision could not be stated to have been seen in this case, and other observations point to the formation of long protoplasmic connexions between the leucocytes of the oyster as a repair phenomenon somewhat equivalent to the clotting effect of blood in vertebrates. The cultivation of the leucocytes of the oyster may nevertheless be possible, since Carrel and Burrows and later workers have shown that even specialised tissues of vertebrates can be grown outside the body in media and under conditions now well-known. A successful cultivation of the leucocytes of the oyster would undoubtedly suggest methods of attacking the problem of the cultivation of human leucocytes outside the body under known conditions and to yield known properties in the leucocytes. The potentialities of human leucocytes cultivated under such conditions may be so great that sufficient excuse is provided for any speculation which may point even faintly to a method by which such a desirable product may be attained. J. H. ORTON.

The Marine Biological Laboratory,

The Hoe, Plymouth, April 28.

\section{Periodical Phenomena in the Temperature Functions of Certain Properties of the Metals.}

IT is well known that the assumption of a quantum distribution of the energy of vibrations of the atoms in solids can be used to explain changes of the specific heat and other properties at low temperatures. Now this quantum distribution also seems to reveal itself in a more direct manner.

I have measured, partly in collaboration with $\mathrm{Mr}$. F. Gunneson, the thermoelectric force at the ordinary temperature of specimens of iron and tungsten that had been successively heated at different temperatures and each time rapidly cooled. The thermoelectric force taken as a function of the heating temperature shows periodical changes, and I could easily distinguish certain repeated intervals of transformation. The approximative absolute temperature, $T_{n}$, of transformation, satisfies the relation $\mathrm{T}_{n}=\mathrm{A} . n$, where $\mathrm{A}$ is a constant and $n$ an integer number. This relation has been verified experimentally for iron from $n=3$ to I2, and for tungsten from $n=4$ to 9. The mean value found for $\mathrm{A}$ was for pure iron $97^{\circ}$ and for tungsten $82^{\circ}$.

As remarked above, this phenomenon is most probably due to the quantum distribution of vibration energy. As the mean number of quanta of the atoms, however, increases continually with the temperature, I have assumed, to explain the periodicities, that the transformations occur at every temperature for which a certain fractional part $(\mathbf{I} / v)$ of the atoms has a number of quanta like, or greater than, a new integer. This assumption gives $\AA=\theta / 3 \log r$, where $\theta(=\beta \nu)$ is the characteristic temperature of the metal in consideration. From this relation $r$ may approximately be calculated, though the values are, like those of $\theta$, rather uncertain. I found for iron $r=4 \cdot 6$, and for tungsten $r=3 \cdot 3$. Thus the critical fractional part is about one-fourth, which seems to be a reasonable value.

Physical Institute, University, Lund.

$$
\text { G. Borelius. }
$$

\section{Observation of Comets.}

Dr. Crommelin in his valuable "Comet Notes," B.A.A. Journal, March I922, p. I98, speaking of Reid's comet (r922 a), says " the comet must have been quite well placed for Northern observers last October and November, being of the 9th magnitude." It seems that Mr. Reid discovered the comet only a short time before it passed out of sight. This in cident reminds us of the fact that a considerable number of these objects must escape observation altogether owing to the want of observers. It is remarkable that English astronomers appear hitherto to have taken little interest in cometary work, and that very few comets have been discovered from this country.

This is a regrettable circumstance. Yet the discovery and observation of comets include a comparatively easy and very attractive field of work open to amateurs with moderately powerful instruments. There are a great number of telescopic observers in the United Kingdom who have the means and the time at their disposal to accomplish valuable work in this department if they would only engage in it in an earnest manner. It is a branch which offers special inducements to amateurs, and holds out a greater prospect of brilliant success than perhaps any other sphere of labour. It is hoped, therefore, that some enthusiasts will turn their practical attention to it, for it is fair to suppose that some of them would like to follow in the footsteps of Messier and Pons, and the equally famous modern discoverers of comets, Barnard and Brooks.

The cometary section of the B.A.A. could not have a more capable director than Dr. Crommelin, and it would strengthen his hands, provide material for his researches, and repay him for his labours if the members of the section notified him of their discovery of one or two comets every year.

44 Egerton Road, Bishopston, Bristol.

W. F. Denning.

\section{A Proposed Laboratory Test of the Theory of Relativity.}

I sHould like to comment on the assumption as to the atomic weight of RaG made by Dr. King in his letter in NATURE of May 6, p. 582. Instead of taking this as known, and calculating the atomic weight of radium by taking account of the a-particle, energy and electron masses, it appears to me that the reverse process would be more justified. It is true that there is an unexplained discrepancy in the difference between the atomic weights of uranium and radium, but we have no reason to doubt the value of the atomic weight of radium obtained by Hönigschmid. As his radium was obtained from Joachimstal pitchblende, which contains practically no thorium, there is no liability to disturbance from the presence of isotopes of radium in his material. On the other hand, the theoretical atomic weight of $\mathrm{RaG}$ was obtained by subtracting the mass of five $a$-particles from the atomic weight of Ra. That the lowest value $(206.046)$ found by Hönigschmid for lead from Morogoro pitchblende agrees so nearly with the theoretical value $(205.93)$, shows only that the amount of original lead present in this material was very small, although, of course, it gave splendid confirmation to the theory of isotopy. We cannot say, however, that we are dealing with pure RaG, even though we know that the amount of admixed isotopes must be very small.

In dealing with such small corrections to the atomic weight as are involved in Dr. King's letter, it thus appears unjustifiable to start off by assuming that the atomic weight of RaG is 206.00 . As Dr. King suggests, however, when the technique of the determination of mass spectra has developed, we shall be independent of admixed isotopes, and the problem will then be put to a rigorous test.

The University, Sheffield. 